\title{
Genetics and genomics converge on the human blood fluke Andy Tait
}

Address: Wellcome Centre for Molecular Parasitology, Faculty of Veterinary Medicine, University of Glasgow, University Place, Glasgow G12 8TA, UK. Email: a.tait@vet.gla.ac.uk

Published: 30 June 2009

Genome Biology 2009, 10:225 (doi:10.1 186/gb-2009-10-6-225)

The electronic version of this article is the complete one and can be

found online at http://genomebiology.com/2009//0/6/225

(C) 2009 BioMed Central Ltd

\begin{abstract}
The construction of a genetic map of the human infective blood fluke (Schistosoma mansoni), coupled with the availability of the genome sequence, offers new approaches for research on this important parasitic worm.
\end{abstract}

Schistosomiasis, or bilharzia, is a human tropical parasitic disease caused by blood-dwelling worms of the genus Schistosoma. Several species infect humans, causing disease in Africa, the Arabian peninsula, China, Indonesia, the Philippines, South America and the Caribbean, but the most prevalent is Schistosoma mansoni in sub-Saharan Africa [1]. The parasite has a complex life cycle, with adult worms mating in the mesenteric plexus in humans and producing eggs that migrate to the intestine, causing much of the pathology. The excreted eggs hatch in water and infect freshwater snails, where they undergo further development into the cercarial stage, which leaves the snail and penetrates the skin of the human host in water.

Some 200 million people are thought to be infected with Schistosoma species worldwide, and although the disease causes relatively few deaths, the level of disability is quite high, with recent revisions of mortality and morbidity suggesting that it represents a significant global burden [2]. Mortality is low compared with diseases such as malaria, but schistosomiasis is nevertheless a major disease burden in some of the poorest communities in the world. It is a somewhat neglected disease. There has been less research than for some other tropical diseases and, until recently, this situation was exacerbated by a lack of molecular and genetic tools for developing new approaches to understanding the pathogen's complex biology, including its interaction with mammalian and mollusc hosts, and new methods of disease control. This situation is now changing, with the recent completion of the genome sequence of $S$. mansoni [3], the availability of microarrays and expressed sequence tags $[4,5]$, the identification of the RNA interference (RNAi) pathway [6], and a genetic map of $S$. mansoni, which is published in this issue by Criscione and colleagues [7]. These developments are very important in stimulating research on this important parasite as they provide the means for gene discovery, analysis of gene function and the identification of genetic loci that determine important traits such as drug resistance, virulence and host specificity.

\section{Schistosoma biology enables defined genetic crosses to be made}

Whereas genetic mapping is relatively well developed in the parasitic protozoa, such as Plasmodium [8], Eimeria [9], Toxoplasma [10] and the trypanosomes [11], and has been exploited to map a variety of important traits, equivalent studies in parasitic worms are rare. To a large extent this has been due to a combination of the lack of genomic resources to develop useful genome-wide markers, the life cycles of this group of parasites, which do not easily allow the genotyping and phenotyping of individual progeny from crosses as there are no vegetative expansions of particular stages, and the lack of suitable laboratory animal hosts or culture systems.

Fortunately, such barriers to genetic analysis do not apply to Schistosoma, as the stages in the snail intermediate host expand asexually, the parasite can infect hamsters, and there is a completed genome sequence. These factors have been exploited in the generation of the genetic map described by Criscione et al. [7]. The biology of $S$. mansoni is the key to being able to use genetic analysis to identify genes determining interesting biological traits. After the adult worms 


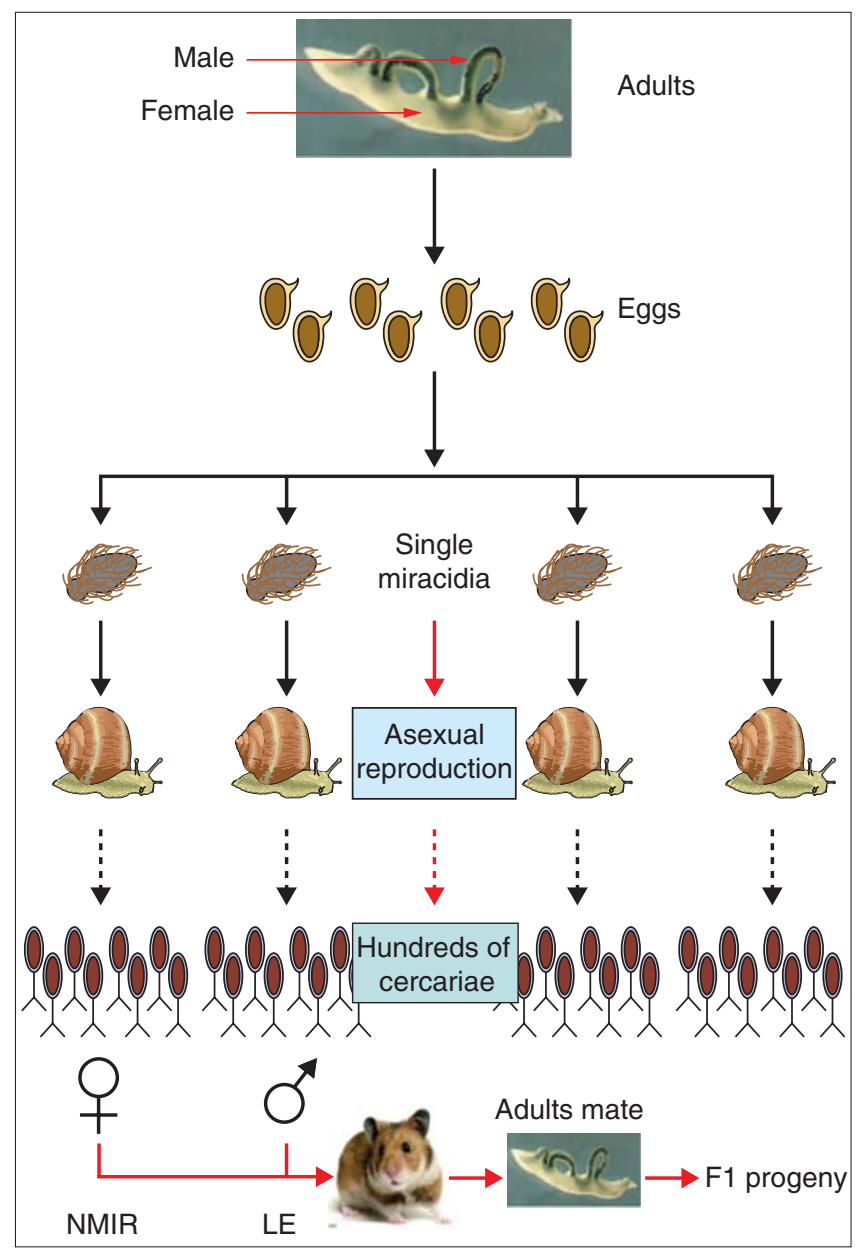

Figure I

The schistosome life cycle and generation of $F$ progeny from strains NMIR and LE. A series of single miracidia from each strain are used to infect individual snails which produce cercariae. These are 'sexed' and then a female from the NMIR strain and a male from the LE strain used to infect a hamster producing adults which mate to generate FI progeny. The F2 generation is produced from the adults by infecting single snails with single FI miracidia derived from the eggs. Male and female FI cercariae are then mixed and used to infect a hamster to produce the F2. Adapted from [I7].

mate, the fertilized eggs are shed in the feces of the infected hamster and develop into motile miracidia on contact with water. Individual miracidia can then be used to infect single snails, in which they develop by clonal expansion into hundreds of genetically identical cercariae, which are induced to leave the snail by exposure to light. The cercariae can then be used to infect a hamster, in which they develop into adult worms (Figure 1). The sex of the parasite is determined before the cercarial stage, so the sex of all the cercariae from a single snail can be ascertained in a small sample, using a sex-specific PCR reaction. This remarkable system allows crosses to be made by co-infecting a hamster with sets of male and female cercariae of different strains. These generate F1 hybrid eggs (Figure 1) that can then be individually expanded into cercariae in snails. From these F1 progeny, one 'clone' of each sex is then used to infect a hamster and generate F2 progeny.

Using the available genome sequence [3], which comprises more than 19,000 scaffolds of the approximately $381-\mathrm{Mb}$ genome, Criscione et al. [7] developed 251 informative polymorphic microsatellite markers and used them to genotype 88 F2 progeny and their parents. The markers were deliberately targeted to different contigs to ensure they were physically separated, although in the case of larger supercontigs, several markers were developed in order to span the whole length. Data on the progeny genotypes were then assembled into linkage groups using the JOINMAP software [12], and gave eight major linkage groups (97\% of markers) with three singletons and two small linkage groups (two to three loci). This correlates well with the known seven pairs of autosomes and one pair of sex chromosomes, with the remaining smaller linkage groups not accounted for. To validate these data and associate each linkage group with a chromosome, fluorescent in situ hybridization (FISH) was undertaken with bacterial artificial chromosome clones or genes representing each chromosome and, with some inconsistencies, seven of the eight linkage groups were successfully mapped to specific chromosomes. The remaining linkage group, number 9, was presumed to represent chromosome 5 .

\section{Features of the $S$. mansoni genetic map}

The final map comprises $1,134.8$ centimorgans (cM) with 210 separate markers and a recombination unit of 227.2 to $244 \mathrm{~kb}$ per $\mathrm{cM}$, a figure comparable to other eukaryotes of similar genome size. From these data, the average distance of a locus from any marker was calculated as $2.9 \mathrm{cM}$ or 683 $\mathrm{kb}$, providing an estimate of the resolution that would be obtained when mapping genes determining a phenotype of interest. A strong positive correlation was observed between the genetic length of each chromosome and its physical size, as determined by cytology. The map will also be a useful tool for assembling the genome sequence by linking contigs in order and identifying inconsistencies in the current assembly, which is particularly important given the highly repetitive nature of the $S$. mansoni genome. This analysis provides evidence that some $57 \%$ of the genome scaffolds are consistent with the map.

Schistosomes have one pair of sex chromosomes (female ZW; male ZZ), with the female being the heterogametic sex in contrast to many higher eukaryotes where the male is heterogametic. Comparison of recombination rates on the autosomes between the two sexes showed that there is a higher recombination rate in females, countering the rule that selection acts against recombination between different sex chromosomes. In addition, the sex chromosomes show a number of unique recombination features, including 
potential hotspots in pseudoautosomal regions of the female chromosome and a region of hemizygosity.

In a cross between the NMRI and LE strains, analysis of segregation ratios for all markers showed agreement with expected Mendelian values, except for two regions on chromosomes 1 and $\mathrm{Z}$, where segregation distortion was observed, with a lower number of NMRI parental alleles than expected. The reasons for this are unclear, but it could be due to selection as a result of incompatibilities between these regions in the two strains, given that the parental strains originate from different geographical regions and have been maintained in the laboratory for more than 40 years.

\section{Potential applications of the genetic map}

A genetic map and the ability to make crosses is an important advance, as it provides another tool in the rapidly developing genetic toolkit for this group of parasitic worms. Although reverse genetic techniques are being rapidly developed for this group of organisms [13], they will always depend on being able to observe a phenotype as a result of the gene disruption. In contrast, forward genetics starts from a phenotype and uses naturally occurring variation. There is a wealth of variant phenotypes to be exploited in this way, and the identification of the genes responsible will provide molecular insight into the biology of the parasite, the immune response it evokes, pathogenesis and mechanisms of drug resistance. Particular examples of such traits include variation in virulence [14], variation in sensitivity to the main anti-schistosome chemotherapeutic, praziquantel [15], and variation in the ability to infect different strains of the snail host [16]. And now such variation is amenable to analysis, additional phenotypes are sure to be identified.

With the current map resolution of approximately $1 \mathrm{Mb}$, genetic analysis of a particular phenotype would only identify loci containing many candidate genes; however, higher resolution could be achieved by generating more progeny and defining higher-density markers provided by single nucleotide polymorphisms (SNPs) from the genome project. Once a locus has been narrowed down to a relatively small number of genes, other approaches can be applied, such as RNAi or transcriptome data, to identify a single gene. A further advantage of the forward genetic approach, now possible using the genetic map developed by Criscione et al. [7], is the ability to investigate phenotypes determined by several loci using quantitative trait analysis. In addition, the availability of genome-wide microsatellite markers will be a powerful resource for addressing a range of questions about the population genetics of the parasite, as well as allowing association studies using field material of defined phenotype. In principal, genetic analysis could also be developed for the related species $S$. japonicum, which is zoonotic (able to be transmitted from animals to humans) and thus raises a series of biological questions about host specificity and whether the parasite populations in different hosts are genetically isolated from each other (host substructuring).

\section{References}

I. Gryseels B, Polman K, Clerinx J, Kestens L: Human schistosomiasis. Lancet 2006, 368:1106-1118.

2. van der Werf MJ, De Vlas SJ, Brooker S, Looman CWN, Nagelkerke NJD, Habbema JDF, Engels D: Quantification of clinical morbidity associated with schistosome infection in sub-Saharan Africa. Acta Trop 2003, 86:125-139.

3. The Schistosoma mansoni genome project [http://www.sanger. ac.uk/Projects/S_mansoni/]

4. Hoffmann KF, Dunne DW: Characterisation of the Schistosoma transcriptome opens up the world of helminth genomics. Genome Biol 2003, 5:203.

5. Schistosoma Genome Network [http://www.nhm.ac.uk/hosted_sites/ schisto/network/]

6. Boyle JP, Wu XJ, Shoemaker CB, Yoshino TP: Using RNA interference to manipulate endogenous gene expression in Schistosoma mansoni sporocysts. Mol Biochem Parasitol 2003, I28:205-2 I5.

7. Criscione CD, Valentim CL, Hirai H, LoVerde PT, Anderson TJ: Genomic linkage map of the human blood fluke Schistosoma mansoni. Genome Biol 2009, I0:R7I.

8. Su X, Ferdig MT, Huang Y, Huynh CQ, Liu A, You J, Wootton JC, Wellems TE: A genetic map and recombination parameters of the human malaria parasite Plasmodium falciparum. Sciencel999, 286:135I-1353.

9. Shirley MW, Harvey DA: A genetic linkage map of the apicomplexan protozoan parasite Eimeria tenella. Genome Res 2000, 10:15871593.

10. Khan A, Taylor S, Su C, Mackey AJ, Boyle J, Cole R, Glover D, Tang T, Paulsen IT, Berriman M, Boothroyd JC, Pfefferkorn ER, Dubey JP, Ajioka JW, Roos DS, Wootton JC, Sibley LD: Composite genome map and recombination parameters derived from three archetypal lineages of Toxoplasma gondii. Nucleic Acids Res 2005, 33:29802992.

II. Cooper A, Tait A, Sweeney L, Tweedie A, Morrison L, Turner CMR, MacLeod A: Genetic analysis of the human infective trypanosome Trypanosoma brucei gambiense: chromosomal segregation, crossing over, and the construction of a genetic map. Genome Biol 2008, 9:RI03.

12. Van Ooijen JW: JoinMap v.4, Software for the calculation of genetic linkage maps in experimental populations. Wageningen, Netherlands: Kyazma BV; 2006.

13. Rinaldi G, Morales ME, Cancela M, Castillo E, Brindley PJ, Tort JF: Development of functional genomic tools in trematodes: RNA interference and luciferase reporter gene activity in Fasciola hepatica. PLoS Negl Trop Dis 2008, 2:e260.

14. Gower CM, Webster JP: Intraspecific competition and evolution of virulence in a parasitic trematode. Evolution 2005, 59:544-553.

15. Fallon PG, Doenhoff MJ: Drug-resistant schistosomiasis: resistance to praziquantel and oxamniquine induced in Schistosoma mansoni in mice is drug specific. Am J Trop Med Hyg 1994, 51:83-88.

16. Webster JP, Davies CM: Coevolution and compatibility in the snailschistosome system. Parasitology 200I, I23:S4I-S56.

17. Hoffman KF, Dunne DW: Characterization of the Schistoma transcriptome opens up the world of helminth genomics. Genome Biol 2003, 5:203. 